OPEN ACCESS

Edited by:

Fatima Baltazar

University of Minho, Portugal

Reviewed by:

Geeta Sikand,

University of California, Irvine

United States

Joan A. Vaccaro,

Florida International University,

United States

*Correspondence:

John Miklavcic

miklavcic@chapman.edu

Specialty section:

This article was submitted to

Clinical Nutrition

a section of the journal

Frontiers in Nutrition

Received: 06 July 2021 Accepted: 03 December 2021 Published: 24 January 2022

Citation:

Dobrow L, Estrada I,

Burkholder-Cooley N and Miklavcic J (2022) Potential Effectiveness of Registered Dietitian Nutritionists in Healthy Behavior Interventions for Managing Type 2 Diabetes in Older Adults: A Systematic Review.

Front. Nutr. 8:737410 doi: 10.3389/fnut.2021.737410

\section{Potential Effectiveness of Registered Dietitian Nutritionists in Healthy Behavior Interventions for Managing Type 2 Diabetes in Older Adults: A Systematic Review}

\author{
Laurel Dobrow ${ }^{1}$, Isabella Estrada ${ }^{1}$, Nasira Burkholder-Cooley ${ }^{1}$ and John Miklavcic ${ }^{1,2 *}$ \\ ${ }^{1}$ Schmid College of Science and Technology, Chapman University, Orange, CA, United States, ${ }^{2}$ School of Pharmacy, \\ Chapman University, Irvine, CA, United States
}

Purpose: A systematic review was conducted to assess how the involvement of a registered dietitian nutritionist (RDN) in healthy behavior interventions (HBIs) potentially affects outcomes in older adults with type 2 diabetes (T2D).

Methods: Literature was searched for primary research published between 2016 and 2020 on $\mathrm{HBI}$ involving a RDN affecting outcomes in older adults with T2D. Evaluations of hemoglobin A1c (HbA1c), blood glucose, blood pressure, cholesterol, anthropometry, body composition, medication usage, healthcare cost, and self-efficacy and/or adherence to healthy behaviors outcomes were selected for inclusion. All the literature included were summarized, evaluated for certainty of evidence criteria, and assessed for bias.

Results: A total of 12 studies were included for assessment. Involvement of a RDN in $\mathrm{HBI}$ was shown to reduce $\mathrm{HbA} 1 \mathrm{c}$, fasting blood glucose, low-density lipoprotein (LDL) cholesterol, and blood pressure and improve lean body mass, body mass index (BMI), and self-efficacy in populations of older adults with T2D. Compared to older adults with $\mathrm{T} 2 \mathrm{D}$ receiving $\mathrm{HBI}$ involving RDNs, patients receiving usual care may incur higher healthcare costs or longer hospital stays. There was a high certainty of evidence for a RDN involvement in $\mathrm{HBI}$ with regard to reduction in $\mathrm{HbA1c}$. There was a moderate certainty of evidence for a RDN involvement in $\mathrm{HBI}$ with regard to favorable changes in weight or body composition and cardiometabolic health outcomes. Statistically significant improvements in outcomes were usually sustained in follow-up after conclusion of $\mathrm{HBI}$.

Conclusion: RDNs may play an integral role in HBls resulting in improved glycemic control, weight management, cardiovascular outcomes, and presumably comorbidity management. RDNs are important facilitators of diet education and nutrition assessment, which are essential in T2D management and should, therefore, be considered for routine inclusion in interprofessional teams for improved outcomes in older adults with T2D.

Keywords: hemoglobin A1c (HbA1c), blood pressure, cholesterol, self-efficacy, medical nutrition therapy (MNT) 


\section{INTRODUCTION}

As of 2018, 26.9 million individuals in the United States have been diagnosed with diabetes with $90-95 \%$ of those patients having type 2 diabetes (T2D) (1). Poorly controlled T2D can lead to the development of various pathologies (2). Comorbidities including hypertension, obesity, dyslipidemia, and peripheral neuropathy contribute to the high societal and economic burden and decreased quality of life in T2D (3). Management of T2D typically consists of education with respect to the pathophysiology of T2D with diet and lifestyle changes that can mitigate T2D-related symptoms. Interventions emphasizing healthy behaviors in $\mathrm{T} 2 \mathrm{D}$ that promote exercise and dietary changes can assist in weight management to positively impact the trajectory of T2D (2), lower hemoglobin A1c (HbA1c) levels, and reduce symptoms associated with comorbid diseases (4). Reduction in fat mass not only lowers individual risk of developing T2D, but also minimizes the impacts of T2Drelated comorbidity such as heart disease (5). Maintaining glycemic control has the potential to reduce complications of T2D including cardiovascular events (4). While the domains of T2D management are within the scope of practice of a registered dietitian nutritionist ( $R D N)$, a summative assessment on the strength of evidence relating intervention with a RDN to specific outcomes in populations with $\mathrm{T} 2 \mathrm{D}$ is needed. This systematic review evaluates the evidence relating healthy behavior intervention (HBI) with a $\mathrm{RDN}$ to outcomes in older adults with T2D.

\section{Demographic of Population With T2D}

The risk of developing T2D increases particularly after the age of 45 years (6). In 2016, 15\% of the United States population was over the age of 65 years and a $7 \%$ increase in this demographic is expected by the year 2040 (7). A similar trajectory is expected on a global scale wherein $\sim 9 \%$ of the global population aged 65 years or older in 2019 is expected to increase to $16 \%$ by 2050 (8). By 2050, $80 \%$ of adults aged 65 years and over in the United States are expected to be experiencing two or more chronic illnesses (9), with an estimated 33\% expected to develop T2D, which is a $23 \%$ increase from current population prevalence (10). An unprecedented increase in the aging population requires tailored interventions delivered by appropriate healthcare professionals to circumvent the impacts associated with increased prevalence of T2D and comorbidity.

\section{Healthy Behavior Intervention}

Healthy behavior intervention is intended to influence individual health behaviors to improve overall health while limiting negative disease-specific outcomes (11). Nutrition counseling, exercise, and engaging in chronic disease-related education are notable elements of HBI that can positively impact health outcomes. HBI constitutes a strategy with high likelihood of positively impacting outcomes in T2D. For example, HBI including group exercise, health education, and goal setting may improve HbA1c, body mass index (BMI), and blood pressure in patients with T2D (12). HBI including a RDN may also improve self-efficacy, adherence to medications, and reduce the burden of comorbidity.

\section{Registered Dietitian Nutritionist Scope of Practice}

Registered dietitian nutritionists utilize an evidence-based approach to prevent or delay disease development and manage acute and chronic disorders (13). Provision of medical nutrition therapy by a $\mathrm{RDN}$ is executed following the nutrition care process consisting of nutrition assessment, diagnosis, intervention, and monitoring and evaluation (13). RDNs are part of a multidisciplinary team and work proactively with patients to develop individualized goals and a care plan appropriate to lifestyle choices, frequency of visits, and existing medical conditions of patient. The nutrition assessment includes a nutrition-focused physical examination where body systems, oral health, muscle wasting, and appetite can be evaluated (13). Relevant tests to determine nutrition status conducted by or ordered by a RDN include blood pressure, weight, height, waist circumference, BMI, skinfold thickness, blood glucose levels, and a blood lipid panel (13). A nutrition care plan may initially involve RDNled nutrition education to the patient that emphasizes the importance of diet prescriptions and why adherence is critical for improved health outcomes. Follow-up nutrition counseling sessions help to guide patients through diet modifications and lifestyle recommendations (13). RDNs also collaborate with case managers, physicians, nurses, pharmacists, speech pathologists, and other health professionals involved in patient care. They counsel patients on food-/nutrientdrug interactions, advise on nutrition-related plans, and are responsible for accounting for prescribed diets, medical foods, dietary supplements, and patient-centered nutrient and energy requirements (13). It is important to note that the comprehensiveness with which the scope of practice is implemented may be limited in some situations, since RDNs provide care in various settings including inpatient, outpatient, community, public, private, and in individual or group environments. RDNs are integral health professionals that provide health and wellness coaching, physical activity counseling, lifestyle advice, and health education as preventive and therapeutic care.

\section{Canadian Diabetes Educator Certification Board (CDECB) and Certified Diabetes Care and Education Specialist (CDCES)}

In the United States, nearly 20,000 healthcare professionals are designated CDCES by the Certification Board for Diabetes Care and Education (CBDCE). Eligibility for the designation includes diabetes education for a minimum of $1,000 \mathrm{~h}$ and at least $15 \mathrm{~h}$ of continuing education related to diabetes (14). Forty-two percent of CDCES-certified professionals are RDNs and $48 \%$ are registered nurses (RNs) (14). Similarly, the CDECB designates the Certified Diabetes Educator (CDE) credential. Providers with CDE credential must be a licensed health professional in Canada, be engaged in a diabetes training program that meets $\mathrm{CDECB}$ standards, 


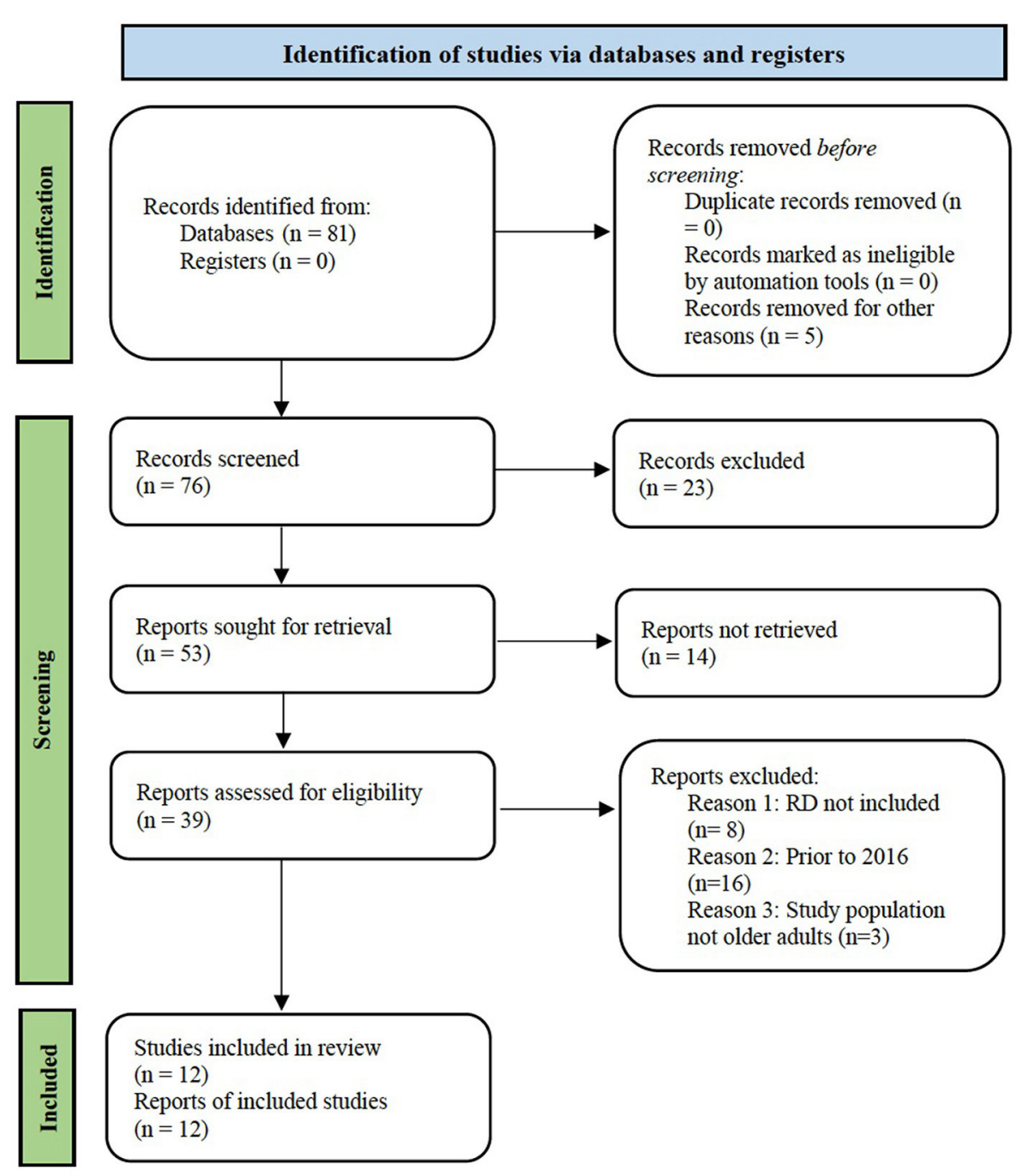

FIGURE 1 | Flowchart of article identification, screening, and selection process for systematic review.

and have a minimum of $800 \mathrm{~h}$ practicum experience (15). As of 2017, there were $\sim 2,200$ CDE providers in Canada (16).

Patients with T2D that engage with the CDE or equivalent have access to more comprehensive T2D education. In a 6month randomized clinical trial, 70 patients receiving T2D education from the $\mathrm{CDE}$ had a greater $\mathrm{HbAlc}$ reduction (9.8 to $8.8 \%$ ) compared to control (9.9-9.3\%) (17). In this regard, it can be concluded that a RDN certified as a diabetes educator has the aptitude to assist a patient with T2D education and improve associated health outcomes. These professionals are largely underutilized, as it is estimated that only $25 \%$ of those diagnosed with T2D in Canada utilize these diabetes education services, with usage even lower among populations aged 65-79 years (18). Therefore, the aim of this systematic review was to determine whether the involvement of a RDN in HBI for older adults with T2D improved glycemic control, body composition, cardiometabolic outcomes, self-efficacy, medication use, and healthcare cost.

\section{METHODS}

Inclusion criteria required a $\mathrm{RDN}$ involvement in $\mathrm{HBI}$ of older adults with T2D. Research studies included in this systematic review were searched and retrieved from GoogleScholar $^{(}$, PubMed $^{\complement}$, and Chapman University Leatherby Libraries $^{\left({ }^{(}\right)}$database on or before December 10, 2020. Search terms for research published from 2016 to 2020 included: "type 2 diabetes or comorbidity," and "older adults," and "diet or registered dietitian or interprofessional team," and "(healthy behavior) intervention or exercise or counseling or medical nutrition therapy (MNT)." Outcomes included HbA1c, BMI, body composition, weight, cholesterol, blood pressure, number of medications, healthcare costs, 
adherence to healthy behaviors, and indicators of self-efficacy (Figure 1).

Study abstracts from search results were screened. Research studies could be excluded by a single author or consensus from two of the authors' review was reached prior to selection for inclusion. Articles were excluded if studies were not interventions, did not include a credentialed RDN in the intervention, or did not have an outcome related to glycemic control, body composition, cardiometabolic outcomes, selfefficacy, medication use, or healthcare cost (Figure 1).

The systematic review followed the preferred reporting items for systematic reviews and meta-analyses (PRISMA) guidelines and included literature was assessed for the level of certainty of evidence following the grading of recommendations assessment, development and evaluation (GRADE) approach considering risk of bias, inconsistency, and indirectness (19). Variability in HBI included components of the intervention, length, frequency and a RDN contact, and range of instruments or surveys used to assess outcomes. As a result, the certainty assessment aimed to inform whether there is a potential effect of a RDN involvement in HBI on a series of outcomes, rather than on certainty of an effect estimate for a given outcome.

A high level of certainty was defined as $>5$ studies, with at least $50 \%$ of the studies showing statistically significant improvement for a particular outcome relative to a usual care group. A moderate level of certainty was defined as 5 or more studies with statistically significant improvement in an outcome over the intervention period for $50 \%$ of HBIs, the improvement was not assessed relative to a comparator group for at least $50 \%$ of studies, or if the total number of studies was only between 2 and 5 and at least $50 \%$ of the studies having statistical significance for a particular outcome. Evidence for all the other combinations of outcomes were deemed as low level of certainty.

\section{RESULTS}

The results of 12 studies meeting the criteria for inclusion of literature in the systematic review were summarized (Table 1).

\section{Hemoglobin A1c and Glycemic Control}

In a study of patients with impaired glycemic control, a HBI including individualized nutrition visits and four RDNled nutrition education sessions resulted in improved HbAlc within group (20). In another study of patients with T2D in Malaysia, participants were randomized to usual care or the Transcultural Diabetes Nutrition Algorithm (tDNA) HBI with meal plan, meal replacements, and at least $150 \mathrm{~min}$ of exercise weekly and either conventional counseling or motivational interviewing involving a RDN (21). After 12 months, participants in the tDNA group had greater decrease in HbAlc and yielded sustained results relative to the usual care group (21). Other studies have examined the role of an extensive lifestyle intervention on variations in HbAlc. In a study of individuals with T2D and a BMI between 25 and $40 \mathrm{~kg} / \mathrm{m}^{2}$ who were not using insulin, participants were randomized to receive moderately intense HBI including counseling every 3 months, a more rigorous HBI consisting of 30-60 min exercise sessions 5-6 times a week, MNT from a RDN, and a pedometer to monitor steps taken with a recommendation to reach at least 10,000 steps/day (22). Those who received the extensive HBI including a RDN exhibited improved glycemic control as indicated by decreased dosage of glucose-lowering medication (22).

Another study analyzed the potential effectiveness of HBI by a RDN in a group, individually, or in a conference call (23). A within-group decrease in $\mathrm{HbAlc}$ occurred in each intervention arm (23). Participants with T2D engaging in a 6-month HBI individually had significantly decreased HbAlc (24). Meeting with a RDN for a structured meal plan or having a weekly phone call with a RDN was shown to significantly reduce HbAlc by $0.61 \%$ (25). Significant reductions in HbAlc were observed from baseline through the extent of the intervention and maintained at 1-year post-intervention for patients with T2D meeting with a RDN (26).

Compared to usual care, another HBI, which included a food workshop and visits with a RDN 3 and 12 months after study enrollment, showed improvement in post-prandial glucose level (27). Although this study did not show a statistically significant difference, the magnitude of change in outcome may have clinical significance (27). Alternatively, a study conducted in Altoona, Blair County, Pennsylvania found that low-to no-cost clinics offering the HBI resulted in statistically significant reductions in HbAlc 1-year post-intervention (28). Other studies examining the impact of extensive lifestyle interventions that emphasize education, exercise, and goal setting drew near identical conclusions (12). HbAlc was reduced by an average of $0.62 \%$ at the end of the intervention and was reduced by another $0.07 \% 1$ year later (12). Regardless of the specific intervention, implementing a HBI under the guidance of a RDN has demonstrated improved glycemic control in older adults with T2D. Hence, their role in an interprofessional healthcare team is vital in the management of T2D.

\section{Body Composition and Weight}

Measurements and assessments of fat mass and fat-free mass are important in the prognosis and monitoring of T2D. A study of patients with T2D not using insulin involved care from a clinical dietitian, lifestyle counseling every 3 months, took part in 30-60 min exercise sessions 5-6 times a week, and instruction to perform at least 10,000 steps/day (22). Compared to the usual care, the HBI group experienced a reduction in BMI, increase in lean muscle mass, and decrease in total body fat (22). Similarly, another study found significant reductions in weight for patients with T2D that completed the diabetes self-management education (DSME) program that involved a RDN (29). Meeting with a RDN for a structured meal plan or having a weekly phone call with a RDN significantly reduced body fat percentage, body weight, and waist circumference (25). When participants took part in a food workshop and regular visits with a RDN, a statistically insignificant reduction in waist circumference and BMI was shown (27). Other research showed similar results, demonstrating a statistically insignificant 
TABLE 1 | Summarized results of studies meeting literature search and review criteria.

\begin{tabular}{|c|c|c|c|c|c|c|c|c|c|}
\hline References & Study population & $\begin{array}{l}\text { Sample } \\
\text { Size }\end{array}$ & Groups & Intervention & Time & Outcomes & Results & $\begin{array}{l}\text { Assessment of } \\
\text { outcome after } \\
\text { intervention }\end{array}$ & Comparison \\
\hline Sbroma et al. (20) & $\begin{array}{l}\text { Diagnosed with } \\
\text { T2D, mean } \mathrm{HbA} 1 \mathrm{c} \\
\text { of } 7.5 \% \text {, average } \\
\text { age of } 59\end{array}$ & 222 & $\begin{array}{l}\text { Intervention } \\
\text { (exercise } \\
\text { physiologist, } \\
\text { endocrinologist, } \\
\text { sports medicine } \\
\text { physician, } \\
\text { psychologist, } \\
\text { RDN, educator, } \\
\text { nurse) }\end{array}$ & $\begin{array}{l}\text { All participants engaged in a } \\
\text { lifestyle intervention after } \\
\text { medical examination and } \\
\text { interview from psychologist. } \\
\text { Intervention included } \\
\text { periodic individualized } \\
\text { nutrition visits and four } \\
\text { RDN-led nutrition education } \\
\text { sessions. Anthropometric } \\
\text { measurements were } \\
\text { compared at baseline, } \\
\text { throughout, and } \\
\text { post-intervention. }\end{array}$ & 3 months & $\begin{array}{l}\text { HbA1c, waist } \\
\text { circumference, } \\
\text { BMl, blood } \\
\text { pressure }\end{array}$ & $\begin{array}{l}\text { HbA1c changed by a mean } \\
\text { value of }-0.6 \pm 1.1 \text {, waist } \\
\text { circumference by }-3.2 \pm \\
4.7 \text {, and BMI by }-0.9 \pm \\
2.50 \text {. Statistically significant } \\
\text { reduction in systolic and } \\
\text { diastolic blood pressure. }\end{array}$ & $\begin{array}{l}24 \text { months follow } \\
\text { up, maintained } \\
\text { improvements in } \\
\text { BMl, weight, waist } \\
\text { circumference, } \\
\text { blood glucose, } \\
\text { HbA1c, blood } \\
\text { pressure. }\end{array}$ & Within group \\
\hline $\begin{array}{l}\text { Gilcharan et al. } \\
\text { (21) }\end{array}$ & $\begin{array}{l}\text { Diagnosed with } \\
\text { T2D and } \\
\text { overweight or } \\
\text { obese BMl }\end{array}$ & 320 & $\begin{array}{l}\text { Intervention (RDN) } \\
\text { vs. usual care }\end{array}$ & $\begin{array}{l}\text { Intervention (tDNA) group } \\
\text { received meal counseling, } \\
\text { exercise plan, and either } \\
\text { counseling with motivational } \\
\text { interviewing or conventional } \\
\text { counseling. Usual care } \\
\text { received dietary and } \\
\text { exercise advice. }\end{array}$ & 6 months & $\begin{array}{l}\text { Eating self-efficacy } \\
\text { measured through } \\
\text { Weight Efficacy } \\
\text { Lifestyle } \\
\text { Questionnaire } \\
\text { (WEL) }\end{array}$ & $\begin{array}{l}\text { WEL scores for the usual } \\
\text { care group was } 121.9 \pm 1.6 \\
\text { at baseline, with } \mathrm{a}-13.2 \pm \\
2.1 \text { difference at } 12 \text { months. } \\
\text { The intervention group who } \\
\text { received conventional } \\
\text { counseling had a baseline } \\
\text { WEL score of } 134.7 \pm 2.6 \text {, } \\
\text { with an } 11.6 \pm 2.6 \text { change } \\
\text { at } 12 \text { months. The } \\
\text { intervention group who } \\
\text { received motivational } \\
\text { interviewing counseling had } \\
\text { a baseline WEL score of } \\
129.1 \pm 2.3 \text {, and a change } \\
\text { of } 28.9 \pm 3.1 \text { at } 12 \text { months. }\end{array}$ & $\begin{array}{l}12 \text { months follow } \\
\text { up, sustained WEL } \\
\text { scores. }\end{array}$ & $\begin{array}{l}\text { Intervention vs. } \\
\text { usual care }\end{array}$ \\
\hline $\begin{array}{l}\text { Johansen et al. } \\
\text { (22) }\end{array}$ & $\begin{array}{l}\text { Diagnosed with } \\
\text { T2D within the } \\
\text { past } 10 \text { years, BMI } \\
25-40 \text {, not using } \\
\text { insulin }\end{array}$ & 98 & $\begin{array}{l}\text { Intervention (RDN, } \\
\text { endocrinologist for } \\
\text { medication } \\
\text { regulation, nurse) } \\
\text { vs. usual care } \\
\text { (nurse and } \\
\text { endocrinologist) }\end{array}$ & $\begin{array}{l}\text { Intervention group received } \\
\text { counseling every } 3 \text { months, } \\
30-60 \text { min exercise } \\
\text { sessions 5-6 times a week, } \\
\text { dietary counseling, and a } \\
\text { pedometer to monitor steps } \\
\text { taken (recommendation to } \\
\text { reach at least 10,000 } \\
\text { steps/day). Usual care } \\
\text { group received T2D } \\
\text { information, lifestyle advice, } \\
\text { and medical counseling } \\
\text { every } 3 \text { months from nurse. }\end{array}$ & 12 months & $\begin{array}{l}\text { HbA1c, changes } \\
\text { in blood glucose } \\
\text { lowering } \\
\text { medication, BMl }\end{array}$ & $\begin{array}{l}\text { HbA1C reduced in the } \\
\text { intervention group from } 6.65 \\
\text { to } 6.34 \%, 6.74 \text { to } 6.66 \% \text { in } \\
\text { the standard care group. } \\
73.5 \% \text { of individuals in the } \\
\text { intervention group were able } \\
\text { to lower their dosages of } \\
\text { glucose lowering } \\
\text { medication, compared to } \\
26.4 \% \text { in the usual care } \\
\text { group. }-2.01 \text { change in } \\
\text { BMl among intervention } \\
\text { group, }-0.69 \text { usual care } \\
\text { group }(p=0.001) \text {. }\end{array}$ & N/A & $\begin{array}{l}\text { Intervention vs. } \\
\text { usual care }\end{array}$ \\
\hline
\end{tabular}


TABLE 1 | Continued

\begin{tabular}{|c|c|c|c|c|c|c|c|c|c|}
\hline References & Study population & $\begin{array}{l}\text { Sample } \\
\text { Size }\end{array}$ & Groups & Intervention & Time & Outcomes & Results & $\begin{array}{l}\text { Assessment of } \\
\text { outcome after } \\
\text { intervention }\end{array}$ & Comparison \\
\hline $\begin{array}{l}\text { Delahanty et al. } \\
\text { (23) }\end{array}$ & $\begin{array}{l}\text { Diagnosed with } \\
\text { T2D, HbA1C } \\
\text { between } 6.5 \text { and } \\
11.5 \text { and } \mathrm{BMl}> \\
25 \mathrm{~kg} / \mathrm{m}^{2}(>23 \\
\mathrm{kg} / \mathrm{m}^{2} \text { if the } \\
\text { participant was } \\
\text { Asian) }\end{array}$ & 208 & Intervention (RDN) & $\begin{array}{l}\text { All participants received } \\
\text { dietary counseling either } \\
\text { from an RDN in a } \\
\text { conference call, in person } \\
\text { with others, or referral to } \\
\text { individual HBI. }\end{array}$ & 12 months & $\begin{array}{l}\text { Cost of each } \\
\text { intervention }\end{array}$ & $\begin{array}{l}\text { Individual HBI was the most } \\
\text { cost effective }(\$ 591) \\
\text { followed by in person } \\
\text { counseling }(\$ 1,380) \text { and } \\
\text { conference call }(\$ 1,814) \text {. }\end{array}$ & N/A & $\begin{array}{l}\text { Intervention } \\
\text { groups }\end{array}$ \\
\hline Mottalib et al. (25) & $\begin{array}{l}\text { Diagnosed with } \\
\text { T2D and } \\
\text { overweight or } \\
\text { obese BMI. Ages } \\
60 \pm 10, \text { not using } \\
\text { insulin but other } \\
\text { T2D medication } \geq \\
3 \text { months }\end{array}$ & 108 & $\begin{array}{l}\text { Intervention A vs. } \\
\text { B vs. C (RDN) }\end{array}$ & $\begin{array}{l}\text { Group A received } \\
\text { individualized dietary } \\
\text { counseling from RDN } \\
\text { regarding eating plan, group } \\
\text { B received individualized } \\
\text { dietary counseling from } \\
\text { RDN regarding meal } \\
\text { planning, group C received } \\
\text { the same intervention as B } \\
\text { but over the phone. }\end{array}$ & 16 weeks & $\begin{array}{l}\text { HbA1c, BMI, waist } \\
\text { circumference }\end{array}$ & $\begin{array}{l}\text { No reduction in } \mathrm{HbA} 1 \mathrm{c} \text { for } \\
\text { group A, but a reduction for } \\
\text { group B }(-0.66) \text { and C } \\
(-0.61) \text {. BMI decreased for } \\
\text { groups A, B, C by }-0.43 \text {, } \\
-1.26,-1.06 \text {, respectively. } \\
\text { Waist circumference }(\mathrm{cm}) \\
\text { decreased most in group B } \\
(-5.0 \mathrm{~cm}) \text { and least in group } \\
\text { A }(-0.4 \mathrm{~cm}) .\end{array}$ & N/A & $\begin{array}{l}\text { Intervention } \\
\text { groups }\end{array}$ \\
\hline $\begin{array}{l}\text { Alonso- } \\
\text { Dominguéz et al. } \\
\text { (27) }\end{array}$ & $\begin{array}{l}25-70 \text { years old } \\
\text { with T2D }\end{array}$ & 204 & $\begin{array}{l}\text { Intervention } \\
\text { (nurse, } \\
\text { smartphone app } \\
\text { developed with } \\
\text { help of RDN and } \\
\text { physical activity } \\
\text { experts) vs. usual } \\
\text { care }\end{array}$ & $\begin{array}{l}\text { Intervention group and usual } \\
\text { care received dietary } \\
\text { counseling. Intervention } \\
\text { engaged in food workshop, } \\
\text { exercise, and received } \\
\text { smartphone application to } \\
\text { assist in adherence to the } \\
\text { Mediterranean diet. }\end{array}$ & 3 months & $\begin{array}{l}\text { Post-prandial } \\
\text { glucose, blood } \\
\text { pressure, waist } \\
\text { circumference, } \\
\text { BMI, adherence to } \\
\text { Mediterranean diet } \\
\text { through } \\
\text { Mediterranean } \\
\text { Diet Adherence } \\
\text { Screener (MEDAS) } \\
\text { questionnaire }\end{array}$ & $\begin{array}{l}\text { Statistically insignificant } \\
\text { reduction in post-prandial } \\
\text { glucose, blood pressure, } \\
\text { waist circumference, and } \\
\text { BMl in the intervention } \\
\text { group at } 12 \text { months follow } \\
\text { up. At } 12 \text { months the } \\
\text { intervention group received } \\
\text { a } 8.4 \text { on the MEDAS, usual } \\
\text { care received } 7.1 \text {. }\end{array}$ & $\begin{array}{l}12 \text { months follow } \\
\text { up, sustained } \\
\text { adherence to } \\
\text { Mediterranean } \\
\text { Diet. }\end{array}$ & $\begin{array}{l}\text { Intervention vs. } \\
\text { usual care }\end{array}$ \\
\hline Agee et al. (28) & $\begin{array}{l}\text { Diagnosed with } \\
\text { T2D >6 months }\end{array}$ & 224 & $\begin{array}{l}\text { Intervention (RDN, } \\
\text { PCP) vs. usual } \\
\text { care. }\end{array}$ & $\begin{array}{l}\text { Intervention group received } \\
\text { HBI from an RDN in addition } \\
\text { to their PCP, usual care } \\
\text { received care from their } \\
\text { PCP. }\end{array}$ & 12 months & $\begin{array}{l}\text { HbA1c, systolic } \\
\text { and diastolic blood } \\
\text { pressure }\end{array}$ & $\begin{array}{l}\text { The intervention group } \\
\text { experienced a mean change } \\
\text { of }-0.8 \% \mathrm{HbA} 1 \mathrm{c}, \mathrm{a}-8.2 \\
\mathrm{mmHg} \text { in systolic blood } \\
\text { pressure, and }-4.3 \mathrm{mmHg} \\
\text { in diastolic blood pressure, } \\
\text { both statistically significant. }\end{array}$ & N/A & $\begin{array}{l}\text { Intervention vs. } \\
\text { usual care }\end{array}$ \\
\hline
\end{tabular}


TABLE 1 | Continued

\begin{tabular}{|c|c|c|c|c|c|c|c|c|c|}
\hline References & Study population & $\begin{array}{l}\text { Sample } \\
\text { Size }\end{array}$ & Groups & Intervention & Time & Outcomes & Results & $\begin{array}{l}\text { Assessment of } \\
\text { outcome after } \\
\text { intervention }\end{array}$ & Comparison \\
\hline Finn et al. (12) & $\begin{array}{l}\text { Recent T2D } \\
\text { diagnosis, }>40 \\
\text { years of age, } 2+ \\
\text { cardiovascular risk } \\
\text { factors }\end{array}$ & 164 & $\begin{array}{l}\text { Intervention (RDN, } \\
\text { nurse, physical } \\
\text { activity specialist, } \\
\text { physician) }\end{array}$ & $\begin{array}{l}\text { All participants were placed } \\
\text { into the intervention group, } \\
\text { all engaged in a } \\
\text { community-based } \\
\text { intervention program with a } \\
\text { multidisciplinary team that } \\
\text { lead group exercise, } \\
\text { individual counseling, and } \\
\text { health related seminars. }\end{array}$ & 16 weeks & $\begin{array}{l}\text { HbA1c targets, } \\
\text { BMI, systolic and } \\
\text { diastolic blood } \\
\text { pressure, LDL, } \\
\text { perceived quality } \\
\text { of life (measured } \\
\text { through EQ-VAS } \\
\text { score) }\end{array}$ & $\begin{array}{l}\text { BMI decreased by a mean } \\
\text { value of } 1.1 \mathrm{~kg} / \mathrm{m}^{2}, \mathrm{HbA} 1 \mathrm{c} \\
\text { targets were met by } 75 \% \text { of } \\
\text { participants compared to } \\
53 \% \text { at baseline. Systolic } \\
\text { blood pressure was } \\
\text { reduced by an average of } \\
8.8 \mathrm{mmHg} \text {, diastolic by } 5.2 \\
\mathrm{mmHg} \text {. Perceived quality of } \\
\text { life increased by a value of } \\
8 . \text { Statistically significant } \\
\text { reduction in total and LDL at } \\
\text { end of intervention and } 1 \\
\text { year. }\end{array}$ & $\begin{array}{l}12 \text { months follow } \\
\text { up, all results } \\
\text { maintained, } \\
\text { physical activity } \\
\text { targets met. }\end{array}$ & Within group \\
\hline $\begin{array}{l}\text { Miller and } \\
\text { Akohoue (30) }\end{array}$ & $\begin{array}{l}\text { African American } \\
\text { women over } 50 \\
\text { years of age with } \\
\text { T2D }\end{array}$ & 12 & $\begin{array}{l}\text { Intervention } \\
\text { (previously with } \\
\text { RDN) }\end{array}$ & $\begin{array}{l}\text { Compared baseline to } \\
\text { post-intervention results. } \\
\text { Participants filled out dietary } \\
\text { self-care questionnaire and } \\
\text { were interviewed. }\end{array}$ & $\begin{array}{l}\mathrm{N} / \mathrm{A}, 2 \text { year follow } \\
\text { up results }\end{array}$ & $\begin{array}{l}\text { HbA1c, systolic } \\
\text { blood pressure, } \\
\text { frequency of } \\
\text { high-fat food } \\
\text { consumption, } \\
\text { spacing out } \\
\text { carbohydrates } \\
\text { throughout the } \\
\text { day, BMl }\end{array}$ & $\begin{array}{l}\text { Reduced } \mathrm{HbA1C} \text {, reduced } \\
\text { systolic blood pressure, } \\
\text { reduced frequency in } \\
\text { fatty-food consumption, and } \\
\text { increased spacing of } \\
\text { carbohydrates throughout } \\
\text { the day. A statistically } \\
\text { significant reduction in } \\
\text { frequency of fruit and } \\
\text { vegetable intake was } \\
\text { observed. Internal factors } \\
\text { such as motivation and } \\
\text { external factors such as } \\
\text { social support were the } \\
\text { most prevalent facilitators } \\
\text { and barriers. Baseline BMl } \\
40.85,2 \text { year follow up } 41.1 .\end{array}$ & $\begin{array}{l}24 \text { months follow } \\
\text { up, reduction in } \\
\text { fruit and vegetable } \\
\text { intake and } \\
\text { increase in BMl. } \\
\text { Maintained } \\
\text { reduced HbA1c } \\
\text { and systolic blood } \\
\text { pressure. } \\
\text { Reduced } \\
\text { frequency of high } \\
\text { fat food } \\
\text { consumption, } \\
\text { increasing in } \\
\text { spacing } \\
\text { carbohydrates } \\
\text { throughout day. }\end{array}$ & Within group \\
\hline
\end{tabular}


TABLE 1 | Continued

\begin{tabular}{|c|c|c|c|c|c|c|c|c|c|}
\hline References & Study population & $\begin{array}{l}\text { Sample } \\
\text { Size }\end{array}$ & Groups & Intervention & Time & Outcomes & Results & $\begin{array}{l}\text { Assessment of } \\
\text { outcome after } \\
\text { intervention }\end{array}$ & Comparison \\
\hline Saleh et al. (33) & $\begin{array}{l}\text { Newly diagnosed } \\
\text { with T2D and }>25 \\
\text { years of age }\end{array}$ & 500 & Intervention (RDN) & $\begin{array}{l}\text { All participants received } 1 \mathrm{~h} \\
\text { T2D education session from } \\
\text { RDN upon enrollment and } \\
\text { engaged in group } \\
\text { discussions regarding T2D } \\
\text { management. }\end{array}$ & 18 months & $\begin{array}{l}\text { T2D knowledge, } \\
\text { self-care } \\
\text { behaviors, and } \\
\text { attitudes } \\
\text { measured through } \\
\text { a } 4 \text { part interviewer } \\
\text { administered } \\
\text { questionnaire }\end{array}$ & $\begin{array}{l}\text { Total knowledge score pre } \\
\text { intervention among male } \\
\text { participants was } 5.26 \pm \\
2.73 \text { and } 5.62 \pm 3.03 \\
\text { among female participants. } \\
\text { Post-intervention total } \\
\text { knowledge score was } 9.12 \\
\pm 2.31 \text { for male } \\
\text { participants, } 8.04 \pm 2.69 \text { for } \\
\text { females. Total attitude } \\
\text { scores pre intervention were } \\
80.30 \pm 6.61 \text { for males, } \\
79.63 \pm 6.47 \text { for females. } \\
\text { Post-intervention total } \\
\text { attitude scores changed to } \\
85.98 \pm 5.86 \text { for males and } \\
85.57 \pm 6.25 \text { for females. } \\
\text { Pre intervention } 8.3,69.2, \\
25.8, \text { and } 86.7 \% \text { of } \\
\text { participants monitored } \\
\text { blood glucose, exercise, } \\
\text { engaged in foot care and } \\
\text { stopped smoking, } \\
\text { respectively, } \\
\text { post-intervention } 67.7 \text {, } \\
85.2,82.8, \text { and } 92.1 \%, \\
\text { respectively. }\end{array}$ & $\mathrm{N} / \mathrm{A}$ & Within group \\
\hline Miklavcic et al. (34) & $\begin{array}{l}\text { Older adults with } \\
\text { T2D and } 2+ \\
\text { chronic conditions }\end{array}$ & 132 & $\begin{array}{l}\text { Intervention (RDN, } \\
\text { RN, program } \\
\text { coordinator) vs. } \\
\text { usual care }\end{array}$ & $\begin{array}{l}\text { Intervention group } \\
\text { experienced three in-home } \\
\text { visits, participated in } \\
\text { monthly group wellness } \\
\text { program, monthly case } \\
\text { conferencing, and care } \\
\text { coordination. }\end{array}$ & 6 months & $\begin{array}{l}\text { Self-efficacy, } \\
\text { self-management, } \\
\text { and cost of } \\
\text { healthcare }\end{array}$ & $\begin{array}{l}\text { No significant differences } \\
\text { across groups over 6-month } \\
\text { period starting from baseline } \\
\text { in self-efficacy, } \\
\text { self-management, or cost of } \\
\text { healthcare. }\end{array}$ & N/A & $\begin{array}{l}\text { Intervention vs. } \\
\text { usual care }\end{array}$ \\
\hline $\begin{array}{l}\text { Markle-Reid et al. } \\
\text { (35) }\end{array}$ & $\begin{array}{l}\text { Community- } \\
\text { dwelling } \\
\text { individuals } 65 \text { and } \\
\text { older diagnosed } \\
\text { with T2D and 2+ } \\
\text { comorbidities }\end{array}$ & 159 & $\begin{array}{l}\text { Intervention (RN, } \\
\text { RDN, program } \\
\text { coordinators, peer } \\
\text { volunteer) vs. } \\
\text { usual care }\end{array}$ & $\begin{array}{l}\text { Intervention group received } \\
\text { up to } 3 \text { in home visits from } \\
\text { RDN and/or RN, group } \\
\text { wellness sessions, care } \\
\text { coordination from nurses, } \\
\text { peer volunteers, and } \\
\text { community partners. Usual } \\
\text { care received support from } \\
\text { DEC/PCN. }\end{array}$ & 6 months & $\begin{array}{l}\text { Self-management } \\
\text { (SDSCA), cost, } \\
\text { self-efficacy } \\
\text { (self-efficacy for } \\
\text { managing chronic } \\
\text { disease scale) }\end{array}$ & $\begin{array}{l}\text { Improvement in } \\
\text { self-management among } \\
\text { intervention group. At } 6 \\
\text { months self-efficacy } 8.27 \pm \\
1.57 \text { for intervention group, } \\
8.05 \pm 1.45 \text { for usual care } \\
(P=0.17) \text {, intervention cost } \\
\text { neutral. }\end{array}$ & $\mathrm{N} / \mathrm{A}$ & $\begin{array}{l}\text { Intervention vs. } \\
\text { usual care }\end{array}$ \\
\hline
\end{tabular}


TABLE 2 | Certainty of evidence assessment.

\begin{tabular}{|c|c|c|c|c|c|c|}
\hline Outcomes & $\begin{array}{l}\text { Certainty level of } \\
\text { evidence }\end{array}$ & $\begin{array}{l}\text { Number of } \\
\text { studies }(n)\end{array}$ & $\begin{array}{c}\text { Statistically } \\
\text { significant } \\
\text { improvement }(n)\end{array}$ & $\begin{array}{l}\text { In how many } \\
\text { studies was the } \\
\text { statistically } \\
\text { significant } \\
\text { improvement } \\
\text { assessed } \\
\text { relative to } \\
\text { comparator } \\
\text { group? (n) }\end{array}$ & $\begin{array}{l}\text { In how many } \\
\text { studies was the } \\
\text { statistically } \\
\text { significant } \\
\text { outcome } \\
\text { measured again } \\
\text { (after } \\
\text { intervention } \\
\text { conclusion)? (n) }\end{array}$ & $\begin{array}{c}\text { In how many } \\
\text { studies was the } \\
\text { statistically } \\
\text { significant } \\
\text { improvement } \\
\text { sustained after } \\
\text { intervention? (n) }\end{array}$ \\
\hline $\mathrm{HbA1c}$ and blood glucose & high & 7 & 4 & 2 & 3 & 2 \\
\hline Body composition, weight & moderate & 6 & 4 & 2 & 3 & 2 \\
\hline Cardiometabolic & moderate & 6 & 4 & 1 & 4 & 4 \\
\hline Self-efficacy, adherence to healthy behaviors & low & 5 & 2 & 2 & 2 & 2 \\
\hline Medications & inconclusive & 1 & 0 & $\mathrm{~N} / \mathrm{A}$ & $\mathrm{N} / \mathrm{A}$ & $\mathrm{N} / \mathrm{A}$ \\
\hline Cost & inconclusive & 3 & 0 & $\mathrm{~N} / \mathrm{A}$ & $\mathrm{N} / \mathrm{A}$ & N/A \\
\hline
\end{tabular}

reduction in weight when meal planning was involved in treatment (21).

Registered dietitian nutritionists involved in HBI that included exercise and T2D education successfully impact anthropometric measures. In a study examining the impact of exercise and education, $97 \%$ of participants were overweight or obese based on BMI, with an average HbAlc of $7.35 \%$ at baseline (12). At the conclusion of intervention, BMI significantly decreased by a mean value of 1.1 in a 1-year HBI and the decrease was sustained 1 year after conclusion of the intervention (12). Nutrition education provided by a RDN within a HBI appears to improve body composition.

\section{Cardiometabolic Outcomes}

Among individuals with poor glycemic control, HBI with a RDN can result in clinically significant improvements in blood triglyceride levels and blood pressure (20). A HBI including a food workshop and regular visits with a RDN yielded reduced systolic blood pressure, though the findings were not statistically significant (27). On the other hand, a study of low-to nocost clinics offering a HBI resulted in statistically significant reductions in systolic blood pressure and diastolic blood pressure after 1 year of intervention (28). In another study of women with $\mathrm{T} 2 \mathrm{D}, \mathrm{HBI}$ including a RDN resulted in reduced systolic blood pressure relative to baseline after 2 years (30). A similar study found statistically significant reductions in systolic blood pressure, diastolic blood pressure, and low-density lipoprotein (LDL) cholesterol, which were maintained 1 year after the conclusion of the intervention (12).

Patients with T2D were randomized in a single-arm preand post-test HBI involving a RDN (24). After 6 months of intervention, high-density lipoprotein (HDL) cholesterol significantly increased and serum tumor necrosis factor significantly decreased (24). The production of proinflammatory proteins is linked with increased risk of developing comorbidity and minimization of these markers by intervention is paramount to abrogating the onset of additional comorbidities in addition to T2D. HBI involving a RDN may improve blood pressure, thus enhancing management of T2D and minimizing associated deleterious outcomes.

\section{Self-Efficacy and Adherence to Healthy Behaviors}

Self-efficacy enables patient confidence in their ability to contribute to the maintenance and improvement of health. This can range from being confident in making healthy dietary choices, knowing how to exercise, and employing weight management strategies. Common attitudes of the elderly toward T2D include "diabetes is genetic, destined, and not a serious complication, let it come;" "diabetes self-care is difficult;" "I do not know what diabetes is;" and "doctors and nurses are important facilitators of self-care management" (31). Furthermore, a study conducted with the Sasak Tribe in Indonesia found improved self-efficacy over the intervention period relative to the control group measured with a self-efficacy questionnaire after attending two 60-min diet education sessions for T2D (32).

In a study of patients with T2D in Malaysia, participants were randomized to usual care or the tDNA HBI involving a RDN (21). Patient self-efficacy perceptions were measured by the Weight Efficacy Lifestyle (WEL) survey, which assessed emotions toward food, ability to control their serving sizes and food choices in various social settings, ability to resist eating when experiencing discomfort, and ability to resist eating when in a positive mood (21). After 12 months, the tDNA groups sustained improvements in "resisting eating when experiencing negative emotions, physical discomfort, and positive activities" the WEL scores compared to the usual care group (21). However, there were no significant differences for "resisting eating when food is available and when there is social pressure" across the two groups (21). The trend showed that patients that had greater decrease in weight and $\mathrm{HbA} 1 \mathrm{c}$ levels yielded sustained results and enhanced self-efficacy. Acquiring the tools and skills needed for weight loss were shown to be potentially effective in developing self-efficacy when culturally appropriate nutrition advice is given in tandem with counseling. Similarly, it was found that a 
HBI with a RDN improved attitude toward T2D and self-care activities relative to baseline. Specifically, there was a statistically significant improvement in knowledge and attitudes toward T2D while participants partook in regular exercise, blood glucose monitoring, foot care, and reduced smoking frequency (33). In summary, such evidence suggests intervention with a RDN that helps to develop self-efficacy will promote management of T2D.

Other studies indicate limited improvements in self-efficacy when patients engage in an HBI involving a RDN. In a study of adults diagnosed with T2D experiencing at least two other selfreported chronic conditions, participants were randomized to a HBI with a RDN or usual care group for 6 months (34). No statistically significant differences in self-efficacy were observed possibly due to the high quality, comprehensive care in the usual group, thus limiting differences in measured outcomes (34). A cohort aged 65 years and above diagnosed with T2D and two or more comorbidities who engaged in a 6-month communitybased HBI did not demonstrate significant improvement in selfefficacy relative to the control group (35). While the connection between a HBI involving a RDN and self-efficacy warrants further research, existing evidence indicating improvements in selfefficacy when engaging a RDN is notable and their involvement in an HBI is important for T2D management.

The adoption of program plans including a diet schedule, medication regimen, self-care, or physical activity has enhanced adherence when a RDN is involved in patient care. A HBI including a food workshop and regular visits with a RDN improved adherence to a Mediterranean diet 3- and 12months post-implementation (27). In members of the Sasak Tribes of Indonesia with $\mathrm{T} 2 \mathrm{D}$, implementing a program with culturally appropriate dietary guidelines resulted in improved diet compliance compared to baseline, assessed by a $24-\mathrm{h}$ recall (32). In another study in which a RDN was involved in the care of older adults with T2D, fatty food consumption decreased from 3.5 to 3 days per week, spacing of carbohydrate consumption throughout the day improved, but there was a reduction in fruit and vegetable intake (30). Furthermore, participation in a HBI involving group exercise sessions, education from an interdisciplinary team, and individualized counseling resulted in physical activity targets being met by $44.7 \%$ of the intervention group participants 1 year after the conclusion of intervention (12). In another cohort study of 162 older adults with T2D advised to meet the American Diabetes Association (ADA) guidelines, $>10 \%$ received care from a RDN, thereby limiting potential adherence to interventions (36). A RDN involved in counseling is advantageous to improving diet and yielding lasting dietary changes that can positively impact disease biomarkers in $\mathrm{T} 2 \mathrm{D}$.

\section{Medication Use}

Adhering to a multimedicine regimen can be tedious and potentially costly for the patient. Intervention with a RDN can reduce the total number of medications a patient will require (37). Patients with T2D and elevated BMI not using insulin were randomized to usual care or a HBI involving a RDN that included exercise and a pedometer (22). The HBI group had improved glycemic control after the 12-month intervention compared to baseline, with $73 \%$ of participants in this group able to reduce the dosage of their blood pressure-lowering medication (22). When compared to the intervention group, only $26 \%$ of those in the usual care group were able to reduce medication dosages, with $44 \%$ requiring an increase in medication (22). Engaging a RDN can improve medication adherence, lower the quantity of medication required by a patient, and subsequently improve health outcomes.

\section{Healthcare Cost}

While the cost of a RDN may offset patients initially, the longterm health benefits to patients who receive intervention with a RDN are cost neutral. As of 2017, \$327 billion was spent on care for patients with diabetes (38). As of 2013, patients aged 55-64 years spent $\sim \$ 85,000$ and patients aged $>65$ years spent over $\$ 54,700$ on T2D-related medical care per lifetime (39). Similarly, in a study analyzing healthcare savings in the HBI groups containing a RDN vs. usual care, the HBI group had an estimated cost savings of $\$ 4,241$ per patient due to reduced length of hospital stay (40). Estimated yearly savings for the HBI groups including intervention and drug prescription costs were $\$ 1,660.60$ per person (40). This study did not find a difference in hospital admissions or change in number of medicines; however, the reduced length of hospital stays resulted in cost reductions for patients in HBI including a RDN (40). A study conducted in Altoona, Blair County, Pennsylvania found that low-to no-cost clinics offering the HBI resulted in statistically significant reductions in $\mathrm{HbA1c}$ and systolic blood pressure and diastolic blood pressure 1 year post-intervention (28). However, a different study found that an intervention pertaining specifically to medication adherence was more cost-effective than other types of HBI (37). Furthermore, it was found that the interventions need to be implemented for over 2 years for improved health and cost benefits (37).

In another study, cost-effectiveness for an individual oneon-one HBI involving a RDN was found to be the most affordable option, at one-half and one-third the costs of group and telephone consult, respectively (23). While intervention by telephone incurred the highest cost, it had the highest incremental cost-effectiveness compared to the individual HBI (23). All three of these interventions were successful in showing that RDNs are cost-effective for improving various anthropometric, biochemical, and cardiovascular measures. Alternatively, other studies conducted in Canada examining the total healthcare cost to a patient associated with engaging a RDN in HBI was equivalent when compared to usual care groups $(34,35)$. In summary, research suggests that participating in a HBI including a RDN does not increase healthcare-associated cost in the management of T2D (35).

\section{Certainty of Evidence}

Two prominent sources of bias were identified in interpreting the accumulation of literature for this systematic review. First, the composition of the intervention team for HBIs may have been multidisciplinary and so attributing effects specifically to a RDN may be challenging. Second, the length of intervention (mean $=7.6$; range $=3-18$ months) may 
influence whether involvement of a RDN in the HBI caused a change in a specific outcome measure. Risk of bias was considered negligible, since all the studies included within or among group statistical analyses. Inconsistency was considered negligible, since primary outcomes assessed had considerations made for covariate analyses. Indirectness was present for all the outcomes. For example, glycemic control could be assessed by HbA1c or blood glucose. Similarly, cardiometabolic outcomes included inflammatory cytokine measures, C-reactive protein, and blood cholesterol.

Certainty of evidence (Table 2) had a high level for a RDN involvement in HBI for older adults with T2D in regard to blood sugar management and reduction in HbAlc. The certainty of evidence (Table 2) had a moderate level for decrease in weight, improvement in body composition, or cardiometabolic health outcomes including LDL cholesterol and pressure. Self-efficacy and adherence to healthy behaviors had a low level for certainty of evidence (Table 2) on whether involvement of a RDN in HBI positively influenced these outcomes consistently in older adults with T2D. Finally, the analysis on certainty of evidence (Table 2) for cost and medication use was inconclusive due to the small number of studies conducted, the heterogeneity of data collection tools used, or the wide range of outcomes within each domain.

\section{DISCUSSION}

Healthy behavior intervention involving a $\mathrm{RDN}$ resulted in reductions in $\mathrm{HbAlc}$ evidenced by statistically significant improvements in 4 out of 7 studies included (Table 2). Similarly, strong evidence supports a decrease in blood pressure, cholesterol, BMI, and weight upon engaging in a HBI involving a RDN (Table 2). There is a low level or sparsity of evidence supporting reductions in medications required or used, cost of care, and increase in HDL cholesterol (Table 2). Few studies were found on such outcomes or limited studies indicated statistically significant improvements.

\section{Challenges to Implementation}

One study highlighted three challenges in expanding healthcare settings for patients with T2D to include a RDN (41). First, the time a diabetes educator was able to spend with a patient appeared to be limited, since educators may also fulfill other roles as nurses, pharmacologists, or clinical nutritionists (41). As such, balancing various job titles resulted in not only less interaction with patients, but also potentially lower quality information sessions and an unclear view of their role within a healthcare practice (41). Second, while diabetes educators may have extensive knowledge with respect to the pathophysiology, proper behavior interventions, and medication that may assist in T2D care, their training in behavior change from a psychological perspective may be limited. This makes recommendations, prescribed lifestyle changes, and information difficult for a provider to convey in a patient friendly manner and for a patient to implement (41). Finally, diabetes educators in this study also emphasized a low number of providers that could provide guidance to patients with T2D, which potentially creates a greater demand for care than providers available (41). In another study where RDNs were interviewed, some expressed that physicians did not refer patients to a RDN when needed due to the primary care provider (PCP) preferring to manage $\mathrm{T} 2 \mathrm{D}$ dietary interventions on their own and perceiving a lack of value for what a RDN can offer (42).

\section{Future Directions}

Registered dietitian nutritionists are vital in interprofessional teams to reduce burnout for involved healthcare professionals. The burnout rate of healthcare professionals can be assessed by the Maslach Burnout Inventory (MBI) (43). In a study analyzing the emotional exhaustion of healthcare professionals in an interprofessional team, it was found that there was a larger threshold until emotional exhaustion among these providers relative to clinicians not working as an interprofessional team (44). Additionally, cognitive behavioral teamwork influenced and predicted clinician-perceived safety of patient (44). This is important because the goal of the interprofessional team is to provide the comprehensive care of patient and ensure that care planning is conducive to all the aspects of their health and management of disease. RDNs in interprofessional teams describe their ability to expand their practice by providing direct nutrition care to patients, deliver health initiatives to the local community, and teach other primary healthcare professionals about nutrition (45). Another study showed that RDNs can work harmoniously in a general practice setting and was even beneficial for the other healthcare professionals. Similarly, it was concluded that collaborative care from a physician, nurse, and a RDN was potentially effective in T2D management and was associated with higher quality care for geriatric patients with T2D in the Middle East (46). This study showed the potential effectiveness of a multidisciplinary team and supports a collaborative approach for older adults with T2D.

Interprofessional teams consist of multiple healthcare professionals including a RDN, PCP, RN, pharmacist, physical or occupational therapists, or other providers that coordinate care planning to address the needs of a patient. The Diabetes Self-management Education and Support (DSMES) program consists of interprofessional healthcare teams that assist nearly one million individuals in the United States per year and provides comprehensive support for patients with T2D through accredited services, many of which are covered by Medicare ${ }^{\mathrm{TM}}$, Medicaid $^{\mathrm{TM}}$, and some private insurance companies $(47,48)$. A controlled clinical trial was conducted where participants in the DSME program, engaged in six 1.5-h education sessions, were provided with an information handbook, given at-home activities, and were provided the opportunity to discuss information with others taking part in the intervention (49). Engagement in the DSMES program has been shown to reduce HbA1c (50).

\section{Limitations}

Outcomes related to quality of life, mental health, depressive symptoms, or anxiety were not included due to the substantial heterogeneity in assessment tools used and are, thus, outside the 
scope of this systematic review. Outcomes related to mortality and burden of comorbidity are also outside the scope of this research. Studies on children or younger adults and older adults engaged in a HBI not involving a RDN were not applicable for the purpose of this systematic review.

\section{CONCLUSION}

Effective HBIs focused on the management of T2D catered to the population of older adults have become increasingly important (51). Engaging with a RDN in the DSMES program or with the CDE or equivalent designation can improve health outcomes including reduced $\mathrm{HbAlc}$, improved cardiometabolic parameters, and decreased fat mass. Many care needs of older adults with T2D can be met when healthcare settings and provider teams implement strategies for engagement of patient with a RDN. Successful approaches to T2D care are multifaceted and a comprehensive team of professionals implementing HBI should consider including a RDN can enhance management of the disease and related comorbidity.

\section{REFERENCES}

1. Center for Disease Control and Prevention. National Diabetes Statistics Report 2020 Estimates of Diabetes and Its Burden in the United States. (2020). Available online at: https://www.cdc.gov/diabetes/pdfs/data/statistics/ national-diabetes-statistics-report.pdf (accessed September 14, 2020).

2. Mayo Clinic. Type 2 Diabetes. (2020). Available online at: https://www. mayoclinic.org/diseases-conditions/type-2-diabetes/diagnosis-treatment/ drc-20351199 (accessed October 9, 2020).

3. Jelinek HF, Osman W, Khandoker A, Khalaf K, Lee S, Almahmeed W, et al. Clinical profiles, comorbidities and complications of type 2 diabetes mellitus in patients from United Arab Emirates. BMJ Open Diabetes Res Care. (2017) 5:e00427. doi: 10.1136/bmjdrc-2017-000427

4. Svensson E, Baggesen LM, Johnsen SP, Pedersen L, Nørrelund H, Buhl ES, et al. Early glycemic control and magnitude of HbalC reduction predict cardiovascular events and mortality: population-based cohort study of 24,752 metformin initiators. Diabetes Care. (2017) 40:800-7. doi: 10.2337/dc16-2271

5. Center for Disease Control and Prevention. Adult Obesity Causes \& Consequences. (2021). Available online at: https://www.cdc.gov/obesity/adult/ causes.html (accessed June 17, 2021).

6. Center for Disease Control and Prevention. Diabetes in Youth. (2019). Available online at: https://www.cdc.gov/diabetes/pdfs/library/DiabetesReport-Card-2019-508.pdf (accessed December 28, 2020).

7. Vespa J, Medina L, Armstrong DM. Demographic Turning Points for the United States: Population Projections for 2020 to 2060. (2018). Available online at: https:/www.census.gov/content/dam/Census/library/publications/ 2020/demo/p25-1144.pdf (accessed November 21, 2020).

8. United Nations. World Population Ageing 2019. (2019). Available online at: https://www.un.org/en/development/desa/population/publications/pdf/ ageing/WorldPopulationAgeing2019-Highlights.pdf (accessed July 1, 2021).

9. Bradley D, Hsueh W. Type 2 diabetes in the elderly: challenges in a unique patient population. $J$ Geriatric Med Gerontol. (2016) 2:14 doi: 10.23937/2469-5858/15 10014

10. Center for Disease Control and Prevention. CDC Newsroom Press Release. (2010). Available online at: https://www.cdc.gov/media/pressrel/ 2010/r101022.html (accessed December 12, 2020).

11. National Research Council Panel on Race, Ethnicity, and Health in Later Life. Behavioral Health Interventions: What Works and Why? (2001). Available online at: https://www.ncbi.nlm.nih.gov/books/NBK25527/ (accessed December 12, 2020).

\section{DATA AVAILABILITY STATEMENT}

Publicly available datasets were analyzed in this study. All data sets used are cited in the references section of this article.

\section{AUTHOR CONTRIBUTIONS}

JM was the lead supervisor, as well as the lead in determining methodology, investigation, formal analysis, conceptualization, writing review and editing, and resources. NB-C provided support in writing review and editing as well as support in investigation. IE and LD contributed to data curation and provided support in formal analysis. LD, IE, and JM contributed to visualization. LD was the lead in writing original draft and IE provided support. All authors contributed to the article and approved the submitted version.

\section{FUNDING}

This study was funded by Chapman University.

12. Finn Y, Gorecka M, Flaherty G, Dunne F, O’Brien T, Crowley J. Evaluation of a community-based cardiovascular prevention program in patients with type 2 diabetes. Am J Health Promotion. (2020) 35:6876. doi: $10.1177 / 0890117120931711$

13. The Academy Quality Management Committee. Academy of nutrition and dietetics: revised 2017 scope of practice for the registered dietitian nutritionist. J Acad Nutr Dietetics. (2018) 118:141-65.

14. CBDCE Channel. Becoming a Certified Diabetes Care and Education Specialist (CDCES). (2020). Available online at: https://www.youtube.com/watch?v= 5s6TSncCarw (accessed July 1, 2020).

15. Certified Board for Diabetes Care and Education. Become Certified. (2020). Available online at: https://www.cdecb.ca/faq/do-i-qualify-to-writethe-examination/ (accessed March 3, 2021).

16. Mansell K, Edmunds K, Guirguis L. Pharmacists' scope of practice: supports for canadians with diabetes. Can J Diabetes. (2017) 41:55862. doi: $10.1016 /$ j.jcjd.2017.08.243

17. Gathu CW, Shabani J, Kunyiha N, Ratansi R. Effect of diabetes selfmanagement education on glycaemic control among type 2 diabetic patients at a family medicine clinic in Kenya: a randomised controlled trial. Afr J Primary Health Care Fam Med. (2018) 10:e1-9. doi: 10.4102/phcfm.v10i1.1762

18. Gucciardi E, Xu C, Vitale M, Lou W, Horodezny S, Dorado L, et al. Evaluating the impact of onsite diabetes education teams in primary care on clinical outcomes. BMC Family Prac. (2020) 21:48. doi: 10.1186/s12875-020-01111-2

19. Preferred Reporting Items for Systematic Reviews and Meta-Analyses. PRISMA Statement. (2021). Available online at: http://www.prisma-statement.org/ PRISMAStatement/Checklist (accessed June 28, 2021).

20. Sbroma Tomaro E, Pippi R, Reginato E, Aiello C, Buratta L, Mazzeschi C, et al. Intensive lifestyle intervention is particularly advantageous in poorly controlled type 2 diabetes. Nutr Metab Cardiovasc Dis. (2017) 27:68894. doi: 10.1016/j.numecd.2017.06.009

21. Gilcharan Singh HK, Chee WS, Hamdy O, Mechanick JI, Lee VK, Barua A, et al. Eating self-efficacy changes in individuals with type 2 diabetes following a structured lifestyle intervention based on the transcultural Diabetes Nutrition Algorithm (tDNA): a secondary analysis of a randomized controlled trial. PLoS ONE. (2020) 15:e0242487. doi: 10.1371/journal.pone.0242487

22. Johansen MY, MacDonald CS, Hansen KB, Karstoft K, Christensen R, Pedersen $\mathrm{M}$, et al. Effect of an intensive lifestyle intervention on glycemic control in patients with type 2 diabetes: a randomized clinical trial. JAMA. (2017) 318:637-46. doi: 10.1001/jama.2017.10169

23. Delahanty LM, Levy DE, Chang Y, Porneala BC, Goldman V, McCarthy J, et al. Effectiveness of lifestyle intervention for type 2 diabetes in primary care: the 
REAL HEALTH-diabetes randomized clinical trial. J General Internal Med. (2020) 35:2637-46. doi: 10.1007/s11606-019-05629-9

24. Sohn C, Yu T, Lee M, Park S, Kim J, Han G, et al. Medical nutrition therapy is associated with decreased inflammation in diabetes patients: a randomized controlled trial. J Acad Nutr Dietetics. (2019) 119:73154. doi: 10.1016/j.jand.2019.06.102

25. Mottalib A, Salsberg V, Mohd-Yusof B-N, Mohamed W, Carolan P, Pober DM, et al. Effects of nutrition therapy on HbAlc and cardiovascular disease risk factors in overweight and obese patients with type 2 diabetes. Nutr J. (2018) 17:42. doi: 10.1186/s12937-0180351-0

26. Marincic PZ, Hardin A, Salazar MV, Scott S, Fan SX, Gaillard PR. Diabetes self-management education and medical nutrition therapy improve patient outcomes: a pilot study documenting the efficacy of registered dietitian nutritionist interventions through retrospective chart review. J Acad Nutr Dietetics. (2017) 117:1254-64. doi: 10.1016/j.jand.2017. 01.023

27. Alonso-Domínguez R, García-Ortiz L, Patino-Alonso MC, Sánchez-Aguadero N, Gómez-Marcos MA, Recio-Rodríguez JI. Effectiveness of a multifactorial intervention in increasing adherence to the mediterranean diet among patients with diabetes mellitus type 2: a controlled and randomized study (EMID study). Nutrients. (2019) 11:162. doi: 10.3390/nu11010162

28. Agee MD, Gates Z, Irwin PM Jr. Effect of medical nutrition therapy for patients with type 2 diabetes in a low-/no-cost clinic: a propensity score-matched cohort study. Diabetes Spectr. (2018) 31:83-9. doi: $10.2337 / \mathrm{ds} 16-0077$

29. Marincic PZ, Salazar MV, Hardin A, Scott S, Fan SX, Gaillard PR, et al. Diabetes self-management education and medical nutrition therapy: a multisite study documenting the efficacy of registered dietitian nutritionist interventions in the management of glycemic control and diabetic dyslipidemia through retrospective chart review. J Acad Nutr Dietetics. (2019) 119:449-63. doi: 10.1016/j.jand.2018.06.303

30. Miller ST, Akohoue SA. Two-year follow-up study of a group-based diabetes medical nutrition therapy and motivational interviewing intervention among African American women. Patient Relat Outcome Meas. (2017) 8:5761. doi: 10.2147/PROM.S125884

31. Tan C, Cheng K, Sum CF, Shew J, Holydard E, Wang W. Perceptions of diabetes self-care management among older singaporeans with type 2 diabetes: a qualitative study. J Nurs Res. (2018) 26:242-9. doi: 10.1097/jnr.0000000000000226

32. Has EM, Aulia A, Kusumaningrum T, Efendi F. Ethnic foods diet program improve self-efficacy and diet compliance among type 2 diabetic patients. $J$ Ners. (2020) 14:155-9. doi: 10.20473/jn.v14i2.16642

33. Saleh F, Afnan F, Ara F, Mumu S, Khan A. Diabetes education, knowledge improvement, attitudes and self-care activities among patients with type 2 diabetes in Bangladesh. Jundishapur J Health Sci. (2016) 9:e36058. doi: 10.17795/jihs-36058

34. Miklavcic JJ, Fraser KD, Ploeg J, Markle-Reid M, Fisher K, Gafni A, et al. Effectiveness of a community program for older adults with type 2 diabetes and multimorbidity: a pragmatic randomized controlled trial. BMC Geriatr. (2020) 20:174. doi: 10.1186/s12877-020-01557-0

35. Markle-Reid M, Ploeg J, Fraser KD, Fisher KA, Bartholomew A, Griffith LE, et al. Community program improves quality of life and self-management in older adults with diabetes mellitus and comorbidity. J Am Geriatr Soc. (2017) 66:263-73. doi: 10.1111 /jgs.15173

36. Katsaridis S, Grammatikopoulou MG, Gkiouras K, Tzimos C, Papageorgiou ST, Markaki AG, et al. Low reported adherence to the 2019 American Diabetes Association nutrition recommendations among patients with type 2 diabetes mellitus, indicating the need for improved nutrition education and diet care. Nutrients. (2020) 12:3516. doi: 10.3390/nu12113516

37. Nerat T, Locatelli I, Kos M. Type 2 diabetes: cost-effectiveness of medication adherence and lifestyle interventions. Patient Prefer Adher. (2016) 10:203949. doi: 10.2147/PPA.S114602

38. Center for Disease Control and Prevention. How Type 2 Diabetes Affects Your Workforce. (2018). Available online at: https://www.cdc.gov/diabetes/ prevention/how-type2-affects-workforce.htm (accessed December 29, 2020).
39. Zhuo X, Zhang P, Hoerger TJ. Lifetime direct medical costs of treating type 2 diabetes and diabetic complications. Am J Prev Med. (2013) 45:25361. doi: 10.1016/j.amepre.2013.04.017

40. Johansson T, Keller S, Sönnichsen AC, Weitgasser R. Cost analysis of a peer support programme for patients with type 2 diabetes: a secondary analysis of a controlled trial. Euro J Public Health. (2017) 27:25661. doi: 10.1093/eurpub/ckw158

41. Preechasuk L, Sriussadaporn P, Likitmaskul S. The obstacles to diabetes self-management education and support from healthcare professionals' perspectives: a nationwide survey. Diabetes Metab Syndr Obesity. (2019) 12:717-27. doi: 10.2147/DMSO.S195916

42. Siopis G, Colagiuri S, Allman-Farinelli M. Dietitians' experiences and perspectives regarding access to and delivery of dietetic services for people with type 2 diabetes mellitus. Heliyon. (2020) 6:e03344. doi: 10.1016/j.heliyon.2020.e03344

43. Williamson K, Lank PM, Cheema N, Hartman N, Lovell EO. Comparing the maslach burnout inventory to other well-being instruments in emergency medicine residents. J Graduate Med Educ. (2018) 10:5326. doi: 10.4300/JGME-D-18-00155.1

44. Welp A, Meier LL, Manser T. The interplay between teamwork, clinicians' emotional exhaustion, and clinician-rated patient safety: a longitudinal study. Crit Care. (2016) 20:110. doi: 10.1186/s13054-016-1282-9

45. Beckingsale L, Fairbairn K, Morris C. Integrating dietitians into primary health care: benefits for patients, dietitians and the general practice team. $J$ Primary Health Care. (2016) 8:372-80. doi: 10.1071/HC16018

46. Johnson JM, Carragher R. Interprofessional collaboration and the care and management of type 2 diabetic patients in the Middle East: a systematic review. J Interprofessional Care. (2018) 32:621-8. doi: 10.1080/13561820.2018.1471052

47. Center for Disease Control and Prevention. Diabetes 2017 Report Card (2017). Available online at: https://www.cdc.gov/diabetes/pdfs/library/ diabetesreportcard2017-508.pdf (accessed June 30, 2021).

48. Center for Disease Control and Prevention. National Standards for Diabetes Self-Management Education and Support. (2018). Available online at: https://www.cdc.gov/diabetes/dsmes-toolkit/standards/index.html (accessed November 28, 2020).

49. Hailu FB, Moen A, Hjortdahl P. Diabetes self-management education (DSME) - effect on knowledge, self-care behavior, and self-efficacy among type 2 diabetes patients in ethiopia: a controlled clinical trial. Diabetes Metab Syndr Obesity. (2019) 12. 2489-99. doi: 10.2147/DMSO.S223123

50. Tshiananga JK, Kocher S, Weber C, Erny-Albrecht K, Berndt K, Neeser, et al. The Effect of nurse-led diabetes self-management education on glycosylated hemoglobin and cardiovascular risk factors. Diabetes Educator. (2011) 38:10823. doi: $10.1177 / 0145721711423978$

51. World Health Organization. Diabetes. (2020). Available online at: https:// www.who.int/news-room/fact-sheets/detail/diabetes (accessed October 18, 2020).

Conflict of Interest: The authors declare that the research was conducted in the absence of any commercial or financial relationships that could be construed as a potential conflict of interest.

Publisher's Note: All claims expressed in this article are solely those of the authors and do not necessarily represent those of their affiliated organizations, or those of the publisher, the editors and the reviewers. Any product that may be evaluated in this article, or claim that may be made by its manufacturer, is not guaranteed or endorsed by the publisher.

Copyright (๐ 2022 Dobrow, Estrada, Burkholder-Cooley and Miklavcic. This is an open-access article distributed under the terms of the Creative Commons Attribution License (CC BY). The use, distribution or reproduction in other forums is permitted, provided the original author(s) and the copyright owner(s) are credited and that the original publication in this journal is cited, in accordance with accepted academic practice. No use, distribution or reproduction is permitted which does not comply with these terms. 